\title{
Application of Cryptand to Diazo/PVA Resists
}

\author{
Masaki Okada, Kieko Harada, Naohide Takamura, Toshihiko Hiaki, Kiyomi Matsuda, \\ Hiroshi Hamana ${ }^{a}$, Shigeru Takahara ${ }^{b}$ and Kazuyuki Sugita ${ }^{b}$ \\ College of Industrial Technology, Nihon University, \\ Chiba 275-8575, JAPAN \\ ${ }^{a}$ Faculty of Engineering, Saitama Institute of Technology, \\ Saitama 369-0293, JAPAN \\ ${ }^{b}$ Faculty of Engineering, Chiba University, \\ Chiba 263-8522 JAPAN
}

\begin{abstract}
Photo- and thermal reaction of $p$-substituted benzene diazo compounds (SBD) with [2.2.2]-cryptand (CRP) was studied by UV spectrum and degradation temperature (D.T.). Diazo cation of $p$-chloro benzene diazo compound (CIBD) was reacted with lone pair electrons of nitrogen atom of CRP by UV spectrum data. When CRP was added, photodecomposition rate of SBD with electron withdrawing-substituent (WSBD) and of SBD with electron donating-substituent (DSBD) were accelerated two and ten times larger than that without CRP, respectively. D.T. of WSBD with CRP in solid were lower than that without CRP, but D.T. of DSBD with CRP was similar to that without CRP. Photodecomposition rate of a diphenylamine-4-diazonium sulfate salt/formaldehyde condensate (DSR) / Polyvinyl alcohol (PVA) resist with CRP was 3 times higher than that without CRP. Diazo/PVA resist with CRP will be improved in sensitivity without decreasing thermal stability.
\end{abstract}

Keywords: photosensitive diazo compound, diazo/PVA resist, cryptand, photodecomposition

\section{Introduction}

The para-substituted benzene diazo compounds (SBD) with electron-donating groups (DSBD) are used as the photo acid generator for micro resists [1], photosensitive emulsions for screen printing resists [2], and UV-fixable thermal recording papers $[3,4]$. SBD with electron withdrawing substituent (WSBD) were used for acid generators for cationic polymerization. 18-Crown-6 (18C6) complexes with benzene diazonium cation improve photo and thermal stability of diazo compounds [5-9]. Thermal decomposition of less expensive $\beta$-cyclodextrin (CD) complexes with diphenylamine-4-diazonium sulfate/formaldehyde condensate / PVA resist (DSR-PVA) was suppressed [10]. The coloring of the DSR/PVA emulsion films was suppressed by inclusion of $18 \mathrm{C} 6$ or PEG [10].

In this report, [2.2.2]-cryptand (CRP) which the inner radius is similar than it of 18C6 was applied to $p$-substituted benzene diazo compounds (SBD). Inclusion compound formation of cryptand in methanol was studied by UV spectrum from absorption spectra. Photo- and thermal degradation was followed by UV spectrum. Degradation temperature D.T. was measured by DTA. Stabilization energy of constitution of complex was calculated by WinMOPAC. Application of CRP to DSR / PVA resist was studied by measurement of photodecomposition.

\section{Experimental}

\subsection{Materials}

The $p$-substituted benzene diazonium tetrafluoroborates (SBD) were precipitated by adding hydrogen tetrafluoroborate to an aqueous solution of the corresponding benzene diazonium chloride prepared from $p$-substituted aniline. The solid 
product was recrystallized from ethanol. The conventional DSR photosensitive diazo resin for screen plate making and PVA emulsion for screen printing were purchased from Murakami Screen K.K. The additives used were, [2.2.2]-cryptand (CRP), boron trifluoride diethyl etherate $\left(\mathrm{BF}_{3} \mathrm{O}\right)$ and potassium chloride, $\mathrm{KCl}$ of extra pure grade.

Concentrations of SBD and inclusion compounds in a methanol were $2.5 \times 10^{-4} \mathrm{~mol} / \mathrm{l}$, $1.25 \times 10^{-3} \sim 1.25 \times 10^{-2} \mathrm{~mol} / 1$, respectively. Solid for thermal analysis was prepared by mixing SBD with cryptand. Coating solutions of the resist were prepared by dissolving $0.6 \mathrm{~g}$ of the diazo compound in $100 \mathrm{~g}$ of the matrix PVA-emulsion in water together with the additives. The resist layers (PVA films) for photodecomposition were coated on to glass plates to a thickness of $15.3 \mu \mathrm{m}$.

\subsection{Measurements}

Thermal decomposition of SBD in methanol was monitored by the ultraviolet (UV) absorbance at $\lambda \max$ under light-shielded conditions. Photodecomposition of SBD in methanol was conducted by irradiation by a Sunhayato Chibi Light Model BOX 1 with fluorescence light (FL-6BL). The incident energy was $2.2 \mathrm{~mW} / \mathrm{cm}^{2}$. The absorbances at $\lambda$ max of UV spectra were measured with a Shimazu UV-1600 PC spectrophotometer. UV spectra of the films were measured with a Shimazu UV 1600 PC. Thermal degradation temperature was determined by TGA in solid measured with Mcscience TG-DTA 2000S Analyzer.

\subsection{Computational method}

All geometry optimizations and calculation were carried out with semi-empirical methods at the PM5 Hamiltonian in WinMOPAC (ver.3.5.la, Fujitsu Limited). For other parameters default values programmed in MOPAC2002 [11].

\section{Results and Discussion}

\subsection{UV spectra of ClBD with CRP}

UV spectra of CIBD in methanol with addition of various concentration of CRP are shown in Fig. 1. It has been found that the maximum absorption wavelength $\left(\lambda_{\max }\right)$ of ClBD ions showed no shift with CRP addition, though the $\lambda_{\max }$ of all the diazo compounds with $18 \mathrm{C} 6$ shifted to shorter wavelength [7]. The $\lambda_{\max }$ of CIBD with CD which include did not shift, either [12]. The inner radius of CRP is reported to be $2.4 \AA$ [13], but the outer size of benzene ring containing hydrogen atoms is 7.37 $\AA$. So CRP can't include the benzene ring of CIBD.

An absorbance of CIBD at $\lambda_{\max } 280 \mathrm{~nm}$ became lower with addition of CRP. When more than 7 times of moles of CRP was added to CIBD, the absorbance of ClBD became constant, and CIBD may be included or react with CRP.

When 1-naphthol-4-sulfhonic acid (NW acid) was added to ClBD, a new absorption appeared at $380 \mathrm{~nm}$ due to an azo dye formed by coupling reaction. However, ClBD with CRP showed lower absorbance at $380 \mathrm{~nm}$ than ClBD without CRP, which indicates that $\mathrm{ClBD}$ reacted with $\mathrm{CRP}$.

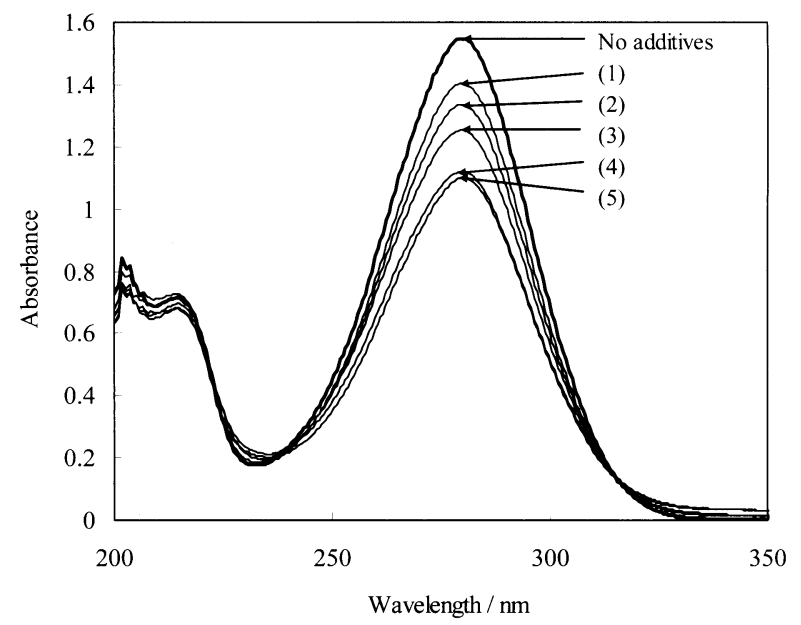

Fig. 1 UV spectra of BD in methanol in the absence and presence of cryptand (CRP) : ClBD, $1 \times 10^{-4} \mathrm{~mol} / \mathrm{L}$, (1) $\mathrm{ClBD}+\mathrm{CRP}\left(2 \times 10^{-4} \mathrm{~mol} / \mathrm{L}\right),(2) \mathrm{ClBD}+\mathrm{CRP}\left(4 \times 10^{-4}\right.$ $\mathrm{mol} / \mathrm{L})$, (3) $\mathrm{ClBD}+\mathrm{CRP}\left(5 \times 10^{-4} \mathrm{~mol} / \mathrm{L}\right)(4) \mathrm{ClBD}+$ $\mathrm{CRP}\left(8 \times 10^{-4} \mathrm{~mol} / \mathrm{L}\right),(5) \mathrm{ClBD}+\mathrm{CRP}\left(10 \times 10^{-4} \mathrm{~mol} / \mathrm{L}\right)$

When ClBD was dissolved in aqueous solution containing $\mathrm{CRP}$ and $\mathrm{BF}_{3} \mathrm{O}$, the absorbance of $\mathrm{ClBD}$ did not change. It was considered that the lone pair electrons of CRP was coordinated by $\mathrm{BF}_{3}$, and an interaction between ClBD and CRP was inhibited.

Similarly, when CIBD was dissolved in an aqueous solution containing an inclusion compound of CRP with $\mathrm{K}^{+}$ion, the absorbance of ClBD did not changed.

\subsection{Photodecomposition of SBD with CRP in} methanol

An influence of CRP addition on the relationship between irradiation time and remaining weight percent of SBD in methanol is shown in Fig. 2. Photodecomposition rate of DSBD with CRP was accelerated more than that without CRP. Photodecomposition rates of $\mathrm{ClBD}$ (WSBD), $\mathrm{BD}$ and 
$\left(\mathrm{C}_{2} \mathrm{H}_{5}\right)_{2} \mathrm{NBD}$ (DSBD) were 2, 3 and 10 times larger than that of SBD without CRP, respectively. The photodecomposition rate of DSBD with CRP was increased more than WSBD with CRP.

The photodecomposition rate of ClBD containing $\mathrm{CRP}$ and $\mathrm{BF}_{3}$ was similar to that of ClBD without additives.

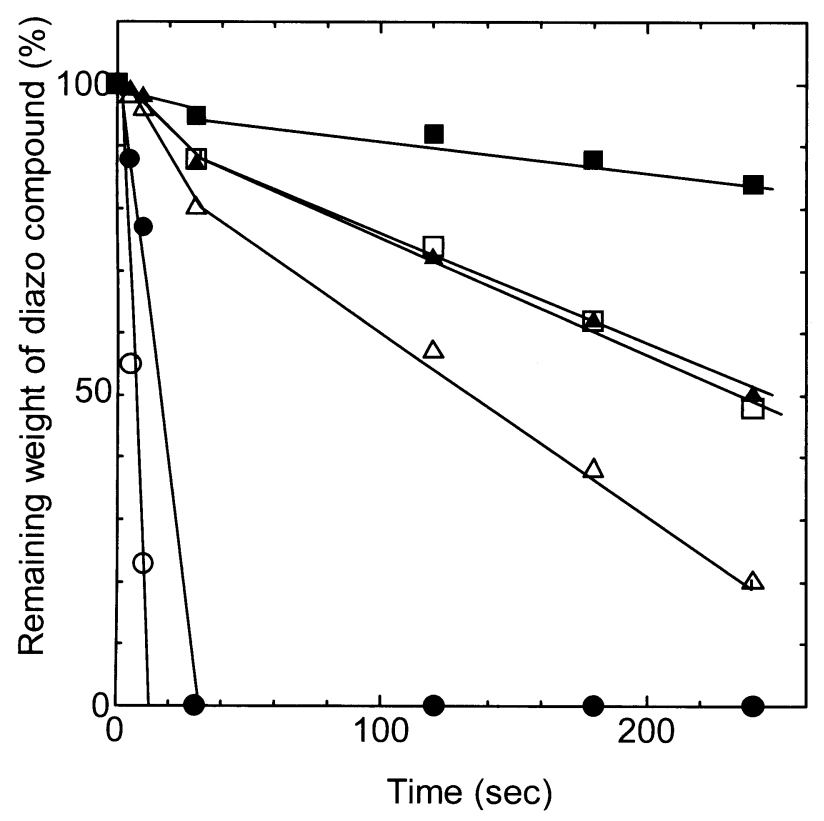

Fig. 2 Photodecomposition of SBD with cryptand (CRP) in methanol, SBD: $5 \times 10^{-5} \mathrm{~mol} / \mathrm{L}, \mathrm{CRP}: 5 \times 10^{-5} \mathrm{~mol} / \mathrm{l}$, Incident energy: $2.2 \mathrm{~mW} / \mathrm{cm}^{2},(\boldsymbol{\square}), \mathrm{ClBD} ;(\square), \mathrm{ClBD}$ + CRP; $(\boldsymbol{\Delta}), \mathrm{BD} ;(\triangle), \mathrm{BD}+\mathrm{CRP} ;(\boldsymbol{O}),\left(\mathrm{C}_{2} \mathrm{H}_{5}\right)_{2} \mathrm{NBD}$; (○), $\left(\mathrm{C}_{2} \mathrm{H}_{5}\right)_{2} \mathrm{NBD}+\mathrm{CRP}$.

\subsection{Thermal decomposition in methanol}

Thermal decomposition of CIBD with CRP in methanol was determined by measuring the intensity, $\lambda_{\max }$ at $283 \mathrm{~nm}$.

Thermal decomposition rate of ClBD with CRP was two times higher than that of CIBD without CRP.

\subsection{Degradation temperature of solid complex}

Degradation temperature, D.T. of SBD with CRP was determined by TGA in solid state.

Relation of D.T. and weight ratio of CRP to ClBD is shown in Fig. 3. The D.T. of CIBD with CRP was lower than that without CRP, and became constant above 0.5 of weight ratio.

Hammett' plots of D.T. in solid state are shown in Fig. 4. The D.T. of SBD with CRP is lower than that without CRP. WSBD with CRP were lower than that with DSBD. It is reported [14] that the electron transfer (by calculation) of SBD from the diazo group to the benzene ring of SBD with electron withdrawing group (WSBD) are easier than that of SBD with electron donating group (DSBD). It is considered the electron poor diazo group $\mathrm{N} \equiv \mathrm{N}^{+}$- of WSBD is easily reacted or included with electron rich cryptand.

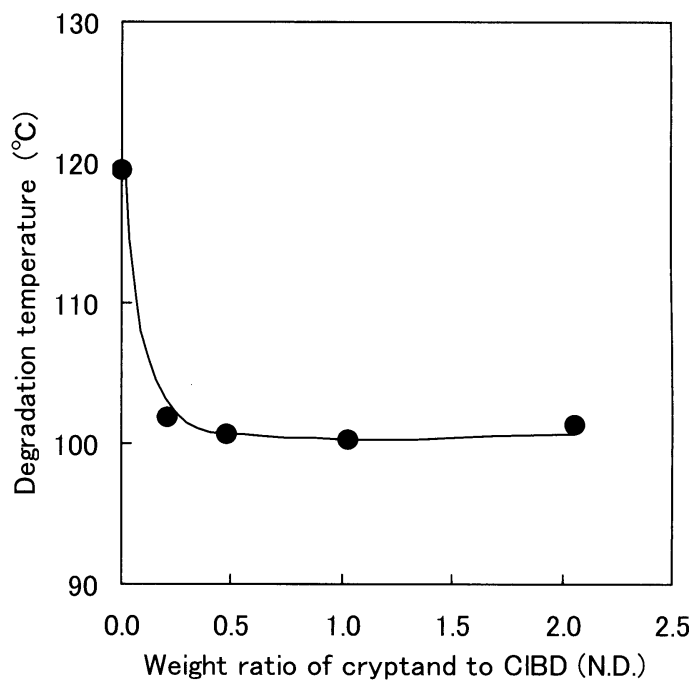

Fig. 3 Relationship between weight ratio of cryptand (CRP) to ClBD and D.T.

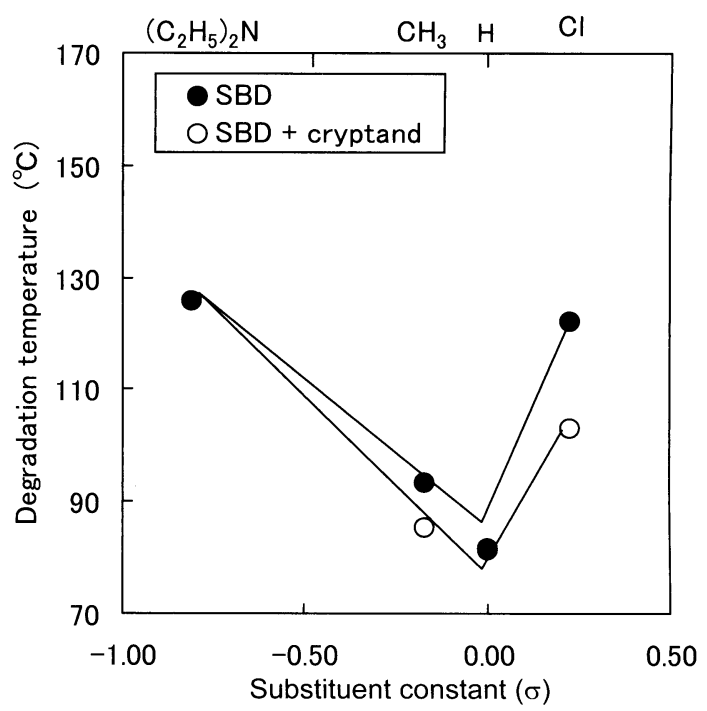

Fig.4 Hammett' plots of D.T. of various $p$-substituted diazo compounds (SBD) with cryptand (CRP) and without cryptand (CRP).

\subsection{Application of CRP to DSR/PVA resists}

The residual DSR with CRP in DSR/PVA film after UV irradiation was measured by UV spectrometry and the remaining weight percent of diazo 
compounds was calculated. The relation of irradiation time and remaining weight percent is shown in Fig. 5. Photodecomposition rate of DSR/PVA film with CRP was accelerated more than that without CRP. When more than $30 \mathrm{wt} \%$ of CRP was added to DSR/PVA resist, photodecomposition rate became constant.

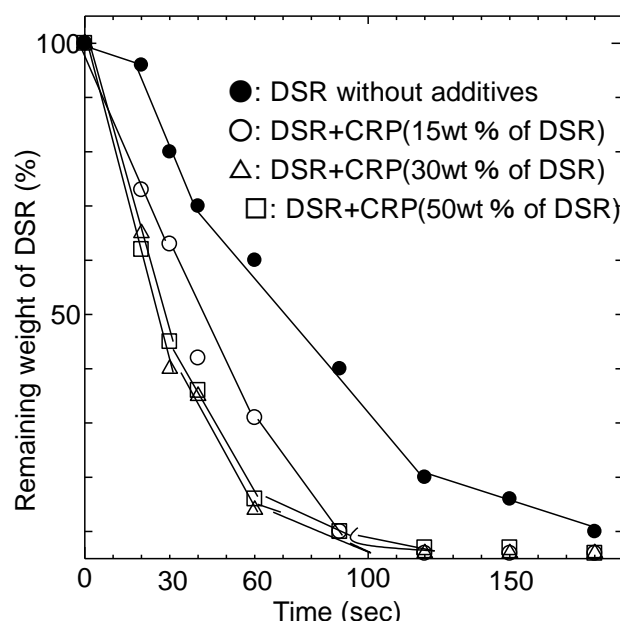

Fig. 5 Photodecomposition of DSR/PVA films with cryptand (CRP). Incident energy : $2.2 \mathrm{~mW} / \mathrm{Cm}^{-2}$

\section{Reaction scheme}

It is calculated that the CRP include $\mathrm{K}^{+}$ion was shown in Fig. 6-A and its stabilization energy is $-62.38 \mathrm{kcal} / \mathrm{mol}$. If CRP include diazo group (Fig.6-B), its stabilization energy is -23.86 $\mathrm{kcal} / \mathrm{mol}$ by WinMOPAC calculation. But photoand thermal decomposition of CIBD with CRP was accelerated. Photodecomposition rate of CIBD in CRP, the lone pair electrons of which coordinate to $\mathrm{BF}_{3}$, did not change. From these results, the diazo cation of ClBD may be react or interact to lone pair electrons of CRP.

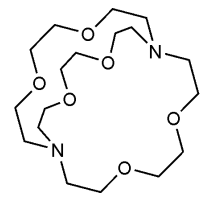

Cryptand (CRP)

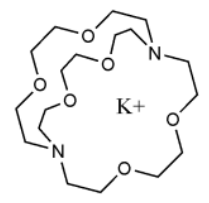

A

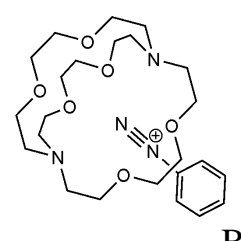

B
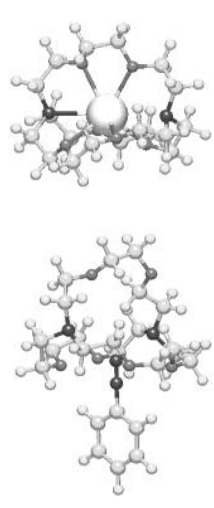

\section{Conclusion}

The D.T. of WSBD was lower than that of DSBD. Electron poor diazo cation easily reacted with CRP. The photodecomposition was accelerated by addition of CRP in methanol, an aqueous solution and the DSR/PVA resist. The diazo/PVA resist with CRP will be improved in sensitivity without decreasing thermal stability.

\section{Acknowledgements}

The authors thank to Mr. Mituru Nagashima and Miss. Machiko Yamaguchi of Nihon University for their helpful suggestion.

\section{References}

1. K.Harada, M.Kushida, K.Saito, K.Sugita and H.Iida, "Micro- and Nanopattering Polymers" H.Ito, E.Reichmanis, O.Naramasu and T.Ueno, eds., ACS Symp. Ser.706, Amer. Chem. Soc., Washington, D.C., 1998, pp. 126-133

2. K.Harada, M.Kushida, K.Saito, K.Sugita and H.Iida, J. Imaging Sci. and Technol., 46, 159 (2002) (1983)

3. T.Usami, T.Tanaka and S.Ishige, Electrophotography, 26, 115 (1987)

4. Y.Yoshida and S.Tatsuta, Electrophotography, 26, 120

5. R.A.Bartsch, H.Chen, N.F.Haddoch and P.N.Juri, J. Am. Chem. Soc., 98, 6753 (1976)

6. T.Kuokkanen and P.O.I.Virtanen, Acta Chem. Scand., ser. B, 33, 725 (1976)

7. K.Harada, K.Sugita and H.Iida, J.Imaging Sci. and Technol., 38, 13 (1994)

8. H.Nakazumi, I.Szele, K.Yoshida and H. Zollinger, Helvetica Chimica ACTA, 66, 1721 Y.Yoshida and S. Tatsuta, Electrophotography, 26, 120 (1987) 9. H.Nakazumi, T. Kitao and H.Zollinger, J. Organic Chem. 52, 2828 (1987)

10. K.Harada, M.Kushida, S.Miyagawa, S.Takahara and K.Sugita, J. Photopolym. Sci. Technol., 18, 187 (2005)

11. J. J. P. Stewart, MOPAC2002 Vl.0, Fujitsu Limited, Tokyo, Japan (2001)

12. K.Fukunishi, J.Hira, H.Yamaoka and M. Nomura, J. Chem. Soc. Perkin Trans. I, p.991 (1985)

13. G. Gokel, " Crown Ethers \& Cryptand" The Royal Society of Chemistry, 1991, p.115

14. T.Tunoda and T.Yamaoka, J.Soc.Photogr.Sci.Eng., 4,331 (1971)

Fig. 6 Inclusion compound model of CRP $+\mathrm{K}^{+}(\mathrm{A})$ and $\mathrm{CRP}+$ benzene diazonium $(\mathrm{BD})(\mathrm{B})$ 